\title{
Genetic analysis of human parainfluenza virus type 4 associated with severe acute respiratory infection in children in Luohe City, Henan Province, China, during 2017-2018
}

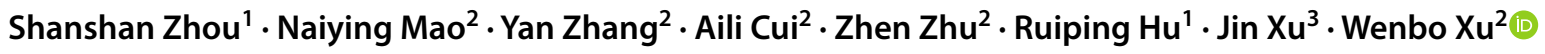

Received: 25 March 2021 / Accepted: 14 May 2021 / Published online: 6 July 2021

(c) The Author(s) 2021

\begin{abstract}
During 2017-2018, nasopharyngeal aspirates (NPAs) from 627 hospitalized patients with severe acute respiratory infection at Luohe Center Hospital were tested by RT-PCR for human parainfluenza virus 4 (HPIV-4). Fourteen (2.2\%) of the 627 samples were positive for HPIV-4. The complete HN gene was amplified from nine positive samples and sequenced. Sequence comparisons showed that the HPIV-4 strains circulating in the city of Luohe are closely related to HPIV-4A strains. Our study indicated that there were multiple lineages of HPIV-4 circulating in Henan Province in China during the study period. This will improve our understanding of the epidemiological and clinical characteristics of HPIV-4.
\end{abstract}

Human parainfluenza viruses (HPIVs), which belong to the family Paramyxoviridae, are enveloped, negative, singlestranded RNA viruses [1,2]. Based on genetic and antigenic variation, HPIVs have been divided into four serotypes in two different genera: Respirovirus (HPIV-1 and HPIV-3) and Orthorubulavirus (HPIV-2 and HPIV-4) [3, 4]. Globally, HPIVs account for a significant proportion of acute respiratory infections (ARIs) among children under the age of 5 years [5, 6]. HPIV-4 was first identified in 1959 by Johnson

Handling Editor: Bert K. Rima.

Shanshan Zhou, Naiying Mao, Ruiping Hu, Jin Xu and Wenbo Xu contributed equally to this work.

\footnotetext{
Ruiping $\mathrm{Hu}$

783674348@qq.com

xujinjc@163.com

$\triangle$ Wenbo Xu

wenbo_xu1@aliyun.com

Shanshan Zhou

zhoushanshan89@sina.com

Naiying Mao

maonaiying@126.com

Yan Zhang

zhangyan9876543@163.com

Aili Cui

cuiaili12345678@163.com
}

$\triangle$ Jin Xu et al. [7] and was first associated with mild respiratory illness in young people. However, recent studies have indicated that it can cause more-severe infections, such as pneumonia and bronchiolitis, in children and elderly individuals [8-12], and in immunocompetent individuals as well as critically ill patients [13-15].

HPIV-4 is subdivided into two subtypes, HPIV-4A and HPIV-4B, based on hemagglutination inhibition and neutralization tests [16]. However, although the number of studies investigating HPIV-4 infection has increased globally, possibly due to improved diagnostic testing procedures, the molecular characteristics of regionally and globally circulating HPIV-4 strains have not been fully elucidated.

\section{Zhen Zhu}

1875818790@qq.com

1 Inner Mongolia Laboratory of Molecular Biology, Inner Mongolia Medical University, Jinshan Avenue, Jinshan Development Zone, Hohhot 010059, Inner Mongolia Autonomous Region, China

2 WHO WPRO Regional Reference Measles/Rubella Laboratory, NHC Key Laboratory of Medical Virology and Viral Diseases (National Institute for Viral Disease Control and Prevention, Chinese Centers for Disease Control and Prevention), 155\# Changbai Road, Changping District, Beijing 102200, China

3 Henan Province Center for Disease Control and Prevention, 105\# Nongye South Road, Zhengzhou 450000, Henan, China 
In this study, 627 nasopharyngeal aspirates (NPAs) were collected from hospitalized patients with severe acute respiratory infection (SARI) in the city of Luohe, Henan Province, China, during 2017-2018 and screened for HPIV-4 infection. Informed consent was obtained from patients or their guardians for the donation of the samples used in this study. This study was approved by the second session of the Ethics Review Committee of the National Institute for Viral Disease Control and Prevention (IVDC) of the China CDC under ethics approval no. IVDC2018 no. 012, and the study was conducted according to the appropriate guidelines. SARI cases were identified through the sentinel surveillance program for hospitalized SARI cases in China (http://www. gov.cn/zwgk/2011-02/11/content_1801649.htm).

The samples were transported to the IVDC of the China $\mathrm{CDC}$ under cold-chain conditions for further analysis. Specimens were stored in sterile minimal medium at -20 or $-80^{\circ} \mathrm{C}$ pending molecular analysis. All procedures were performed in accordance with the relevant guidelines and regulations.

Viral RNA was extracted from NPA samples using a QIAamp Viral RNA Mini Kit (QIAGEN, Valencia, CA, USA), and HPIV-4-positive samples were screened by one-step real-time RT-PCR using a PrimeScript ${ }^{\mathrm{TM}}$ RTPCR Kit (Takara Biotechnology Dalian, China, catalog no. DRR064A) as described previously [17]. The complete hemagglutinin-neuraminidase (HN) gene (1740 nt) of HPIV-4 was amplified by nested RT-PCR for genotyping HPIV-4. The first round of RT-PCR was performed using the following in-house-designed primer pair: forward primer, 5' - ATAGGGGGGAACRCACTTCTCAGC-3'; reverse primer, 5'- GGCRGRTTGTTTRTYGAGGACC-3'. The nested PCR was carried out with the following inner primers: forward primer, 5'-AACAATCCAGARRGACRTCAC ATCAA-3'; reverse primer, 5' - TCTTTCAGTGGATGG TTGAGGA-3'. The RT-PCR products were purified for sequencing using a QIAquick Gel Extraction Kit (QIAGEN, Hilden, Germany), and the amplicons were sequenced on an ABI PRISM 3100 DNA Sequencer (PerkinElmer, Beijing, China). Nucleotide sequences were assembled and edited using Sequencher (Gene Codes Corporation, Ann Arbor, MI, USA). The sequences of HPIV-4 obtained in our study have been submitted to the GenBank database (accession numbers MT681670-MT681678).

Pairwise distances of nucleotide and deduced amino acid sequences were determined by alignment of with 43 HPIV-4 sequences from GenBank using the ClustalW algorithm implemented in MEGA software version 7.0. Phylogenetic analysis based on complete $\mathrm{HN}$ gene nucleotide sequences were performed in MEGA version 7.0 by the maximum-likelihood method using the Kimura 2-parameter substitution model, with statistical significance of phylogenies estimated by bootstrap analysis with 1,000 replicates.
From October 2017 to December 2018, a total of 627 NPAs from inpatients (391 males and 236 females) who met the SARI case definition were collected from Luohe Central Hospital in Henan Province. The hospitalized patients with SARI ranged in age from 0 to 91 years old, with children under 6 years old accounting for $78.0 \%$ (489/627). The median age was 7.9 years.

Of the 627 patients, $14(2.2 \%, 95 \%$ CI 1.1-3.4) were positive for HPIV-4 by real-time RT-PCR. The cycle threshold (CT) values for the HPIV-4-positive samples ranged from 25 to 35 . Most of the CT values of the positive samples ranged from 29 to 33. The ratio of males to females was 1.7:1. Among the 14 patients (median, 2.5 years) with determined HPIV-4, all of them were younger than 7 years old, and $50 \%$ were 0 to 3 years of age (Table 1). Among the pediatric patients, $10(71.4 \%)$ were diagnosed with bronchopneumonia, three $(21.4 \%)$ with bronchiolitis, and one $(7.1 \%)$ with mucocutaneous lymph node syndrome (MCLS).

The seasonality of HPIV-4 was observed throughout the study period. The positive rates of HPIV-4 in spring, summer, autumn, and winter were $1.2 \%$ (1/85), $17.5 \%$ (10/57), $0.5 \%(1 / 199)$, and $0.7 \%$ (2/286), respectively (Fig. 1). The highest detection rates of HPIV-4 were found in summer, and the detection rate of HPIV-4 varied significantly between seasons $\left(\chi^{2}=67.456, P=0.000\right)$.

The complete HN gene sequences of nine HPIV-4 isolates (CT values between 25 and 33) amplified from 14 HPIV4-positive specimens were analyzed. The HN gene failed to be amplified from the remaining five HPIV-4-positive specimens, which had CT values ranging from 27 to 35 in the real-time RT-PCR. This could be due to mismatches in the primer-binding sites or low viral load.

All HPIV-4 HN gene sequences available in the GenBank database (43 sequences) were downloaded and aligned with the nine sequences obtained in this study. Among the nine HPIV-4 HN gene sequences, seven clustered with the

Table 1 Sex and age distribution of HPIV-4-positive patients

$\begin{array}{ll}\begin{array}{l}\text { Total number of } \\ \text { specimens }\end{array} & \begin{array}{l}\text { Number posi- } \\ \text { tive for HPIV-4 } \\ (\%)\end{array} \\ & \end{array}$

\begin{tabular}{lll}
\hline Sex & & \\
Male & 391 & $8(2.0 \%)$ \\
Female & 236 & $6(2.5 \%)$ \\
Age group (years) & & \\
$<1$ & 77 & $1(1.3 \%)$ \\
$\geq 1$ to $<3$ & 238 & $7(2.9 \%)$ \\
$\geq 3$ to $<6$ & 174 & $5(2.9 \%)$ \\
$\geq 6$ to $<14$ & 67 & $1(1.5 \%)$ \\
$\geq 14$ & 71 & 0 \\
Total & 627 & $14(2.2 \%)$ \\
\hline
\end{tabular}


Fig. 1 Monthly distribution of human parainfluenza virus 4 (HPIV-4) infections

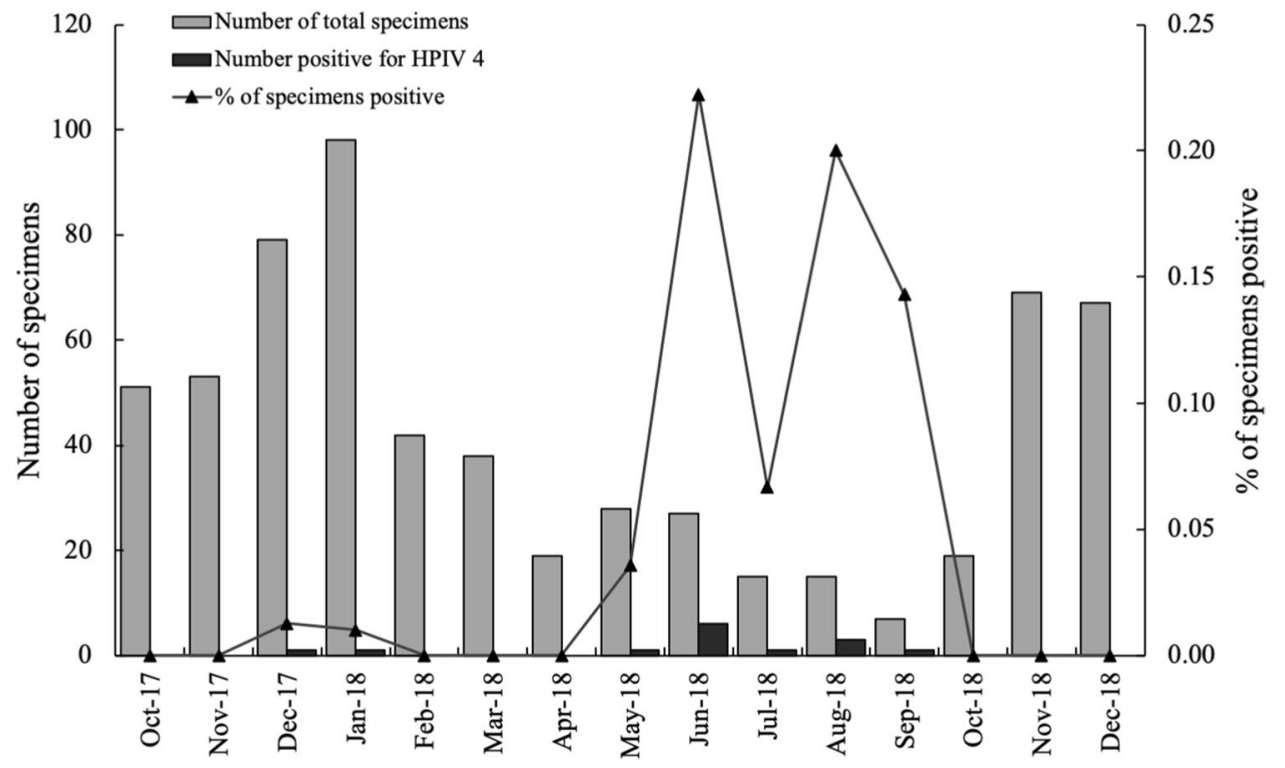

HPIV-4A reference strains, and the remaining two clustered with the HPIV-4B reference strains (Fig. 2). By convention, the prototype of HPIV-4, which was isolated in Japan in 1959 , formed a single lineage, designated as cluster I. The strains from Denmark in 2013, Japan in 2010, and Australia in 2008 constituted cluster II, with a mean nucleotide sequence divergence of $1.7 \%$. Most of the viruses from Asia, with a mean nucleotide sequence divergence of $1.7 \%$, were clustered together and designated as cluster III. The mean distance between the three clusters was $5.4 \%$, which is larger than the mean distance within the three clusters $(1.7 \%)$, in reference to a report that described phylogenetic analysis of HPIV-3 [18]. Cluster III strains were further grouped into four lineages (lineages 1, 2, 3 and 4) with 1.8-2.7\% nucleotide sequence divergence. In our study, six HPIV-4A strains belonged to lineage 4 , together with four previously reported strains from Japan. The remaining HPIV-4A strain was placed into lineage 3 with seven strains from Japan. In addition, two strains from this study were identified as HPIV-4B, which has rarely been reported in recent decades.

The divergence between the seven HPIV-4A strains from our study and the prototype strain M-25 was approximately $6.6 \%-6.9 \%$ and $10.1 \%-11.0 \%$ in the nucleotide and amino acid sequences, respectively. The two HPIV-4B isolates exhibited $4.9 \%-5.0 \%$ nucleotide sequence divergence and 8.0\%-8.2\% amino acid sequence divergence from the prototype strain. Notably, the length of the HN gene sequence (1740 nt) in our nine strains is longer than that of the prototype strain (1725 nt) by 15 nucleotides (5 amino acids) at the carboxy terminus. Compared with other strains, the divergence of nucleotides and amino acids among HPIV4A was $1.0 \%-3.7 \%$ and $0.3-3.4 \%$, respectively. Among the HPIV-4B isolates, it was $0.5-4.9 \%$ and $0.5-8.4 \%$, respectively. Briefly, there are two common subtypes of HPIV-4 in
China, HPIV-4A and HPIV-4B, of which HPIV-4A includes two lineages of cluster III.

In the present study, 14 samples that were positive for HPIV-4 from 627 (2.2\%) SARI patients, collected between October 2017 and December 2018, were analyzed to determine the epidemiology of HPIV-4 in the city of Luohe, Henan Province, China, using q-RT-PCR. All patients with HPIV-4 infection were less than 7 years old, and $57 \%$ of them were younger than 3 years old, indicating that children in this age group are the main HPIV-4-susceptible population. This is in agreement with previous reports. The positive rate of HPIV-4 was $1.2 \%$ among 0.5 -month- to 16 -year-old patients, with the highest prevalence in 3- to 5-year-old children in Beijing, China. The median patient age in Colorado between 2009 and 2012 was 4.1 years old [19, 20]. Therefore, HPIV-4 may be gradually becoming an important cause of SARI in children, with higher severity than previously thought.

In our study, HPIV-4 infections occurred during summer and autumn, especially in summer, which is inconsistent with published studies from other countries. In Japan, Abiko et al. [8] described an outbreak of HPIV-4 infections during the 2011-2012 winter season. A similar result was reported in Canada during 2004-2005 [21]. This may be attributed to different geographical regions and study periods. However, as the number of positive samples in this study was limited, a large-scale investigation of the HPIV-4-positive rate is needed to understand the seasonal patterns of HPIV-4. In addition, considering that only strains from hospitalized patients were analyzed in this study, the possibility that other underestimated strains could be circulating in the general population cannot be ruled out.

In previous studies, HPIV-4 strains were detected at different times in different regions [21-23]. In our study, 
Fig. 2 Phylogenetic tree based on complete HN gene nucleotide sequences of HPIV-4 strains

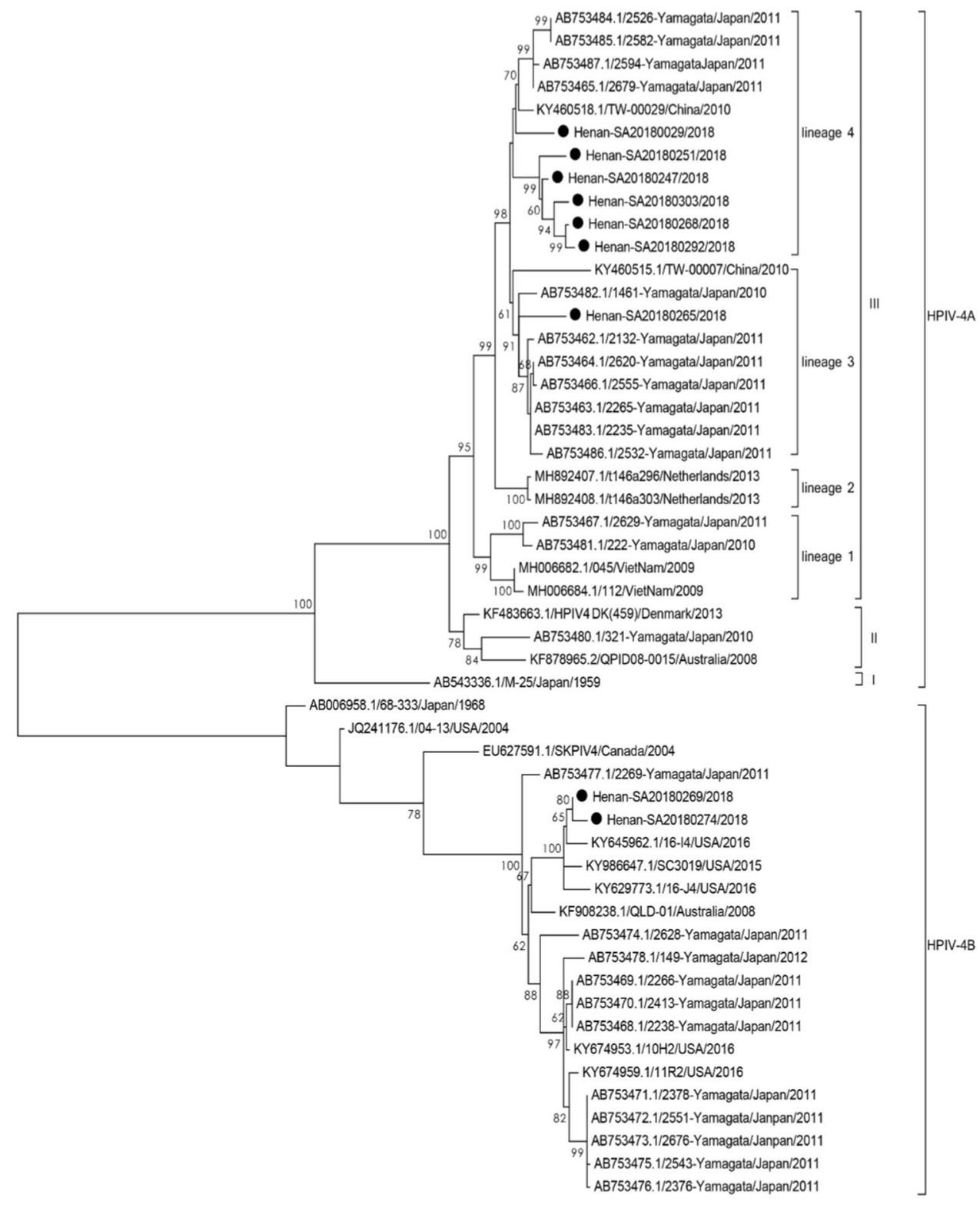

0.02

we characterized the HN gene from HPIV-4-positive samples from patients with SARI and further divided all of the HPIV-4A strains into three clusters. This is the first report describing the phylogeny of HPIV-4 based on complete HN sequences. Compared with the HPIV-4 prototype strain M-25, nine strains in our study, including other strains from GenBank, demonstrated significantly different characteristics. A five-amino-acid insertion in the HN protein of the Luohe strains may result in changes in the antigen-binding site and influence viral replication and transmission. This finding also indicates that the viruses are probably evolving.

Phylogenetic analysis revealed that five (HenanSA20180251/247/303/268/292) of the seven HPIV-4A strains might share a chain of transmission. The remaining two HPIV-4A strains were similar to Japanese strains from 2010 and 2011. Phylogenetic analysis suggested that the domestic HPIV-4A strains belong to lineage 4 of cluster III, which seems to be prevalent. Moreover, two HPIV-4B strains were associated with three USA HPIV-4B strains isolated during 2015-2016. This reveals that the HPIV-4 strains were circulating globally and are more closely related to HPIV-4A than HPIV-4B, based on the HN gene sequences in our study. However, the genetic analysis of HPIV-4 in our study is only representative of Henan province, and not the entire country. In the future, additional sequences are needed to expand the dataset, and additional 
studies are required to determine the relative importance of HPIV-4A and HPIV-4B.

In conclusion, we have reported the $\mathrm{HN}$ gene sequences of nine HPIV-4 strains isolated from SARI patients. The divergence among HPIV-4 strains indicated that these viruses have circulated in the environment for many years and have undergone evolution. To better recognize its clinical importance and seasonal patterns, HPIV-4 should be included in the panels used for routine respiratory virus detection, although most clinical laboratories currently do not screen for HPIV-4 [23, 24]. This report provides valuable information about HPIV-4 isolates that might help to prevent HPIV-related respiratory diseases.

Supplementary Information The online version contains supplementary material available at https://doi.org/10.1007/s00705-021-05154-3.

Acknowledgements The authors are grateful to the clinicians of Luohe Central Hospital for assisting with sample collection.

Author contributions SSZ, NYM and WBX prepared the manuscript. RPH, JX and WBX designed and coordinated the study. YZ, ALC and $\mathrm{ZZ}$ collected the specimens and performed the experiments. SSZ, NYM, YZ, ALC, ZZ and WBX performed the data analysis. All authors read and approved the final manuscript.

Funding Funding was obtained from the Key Technologies R\&D Program of the National Ministry of Science (Grant nos. 2018ZX10713002 and 2018ZX10713001-003) and National Health Commission (Grant no. 131031103000180004).

Data availability All data included in this study are available upon request from the corresponding author.

\section{Declarations}

Conflict of interest The authors declare that they have no competing interests.

Open Access This article is licensed under a Creative Commons Attribution 4.0 International License, which permits use, sharing, adaptation, distribution and reproduction in any medium or format, as long as you give appropriate credit to the original author(s) and the source, provide a link to the Creative Commons licence, and indicate if changes were made. The images or other third party material in this article are included in the article's Creative Commons licence, unless indicated otherwise in a credit line to the material. If material is not included in the article's Creative Commons licence and your intended use is not permitted by statutory regulation or exceeds the permitted use, you will need to obtain permission directly from the copyright holder. To view a copy of this licence, visit http://creativecommons.org/licenses/by/4.0/.

\section{References}

1. Vainionpaa R, Hyypia T (1994) Biology of parainfluenza viruses. Clin Microbiol Rev 7:265-275

2. Branche AR, Falsey AR (2016) Parainfluenza virus infection. Semin Respir Crit Care Med 37:538-554

3. Henrickson KJ (2003) Parainfluenza viruses. Clin Microbiol Rev $16: 242-264$
4. Park KS, Yang MH, Lee CK, Song KJ (2014) Genetic analysis of human parainfluenza viruses circulating in Korea, 2006. J Med Virol 86:1041-1047

5. Pecchini R, Berezin EN, Souza MC, de Andrade V-d-L, Sato N, Salgado $M$ et al (2015) Parainfluenza virus as a cause of acute respiratory infection in hospitalized children. Braz J Infect Dis 19:358-362

6. Weinberg GA (2006) Parainfluenza viruses: an underappreciated cause of pediatric respiratory morbidity. Pediatr Infect Dis J $25: 447-448$

7. Johnson KM, Chanock RM, Cook MK, Huebner RJ (1960) Studies of a new human hemadsorption virus. I. Isolation, properties and characterization. Am J Hyg 71:81-92

8. Abiko C, Mizuta K, Aoki Y, Ikeda T, Itagaki T, Noda M et al (2013) An outbreak of parainfluenza virus type 4 infections among children with acute respiratory infections during the 2011-2012 winter season in Yamagata, Japan. Jpn J Infect Dis 66:76-78

9. Fairchok MP, Martin ET, Kuypers J, Englund JA (2011) A prospective study of parainfluenza virus type 4 infections in children attending daycare. Pediatr Infect Dis J 30:714-716

10. Russell E, Yang A, Tardrew S, Ison MG (2019) Parainfluenza virus in hospitalized adults: a 7-year retrospective study. Clin Infect Dis 68:298-305

11. Steffens A, Finelli L, Whitaker B, Fowlkes A (2016) Populationbased surveillance for medically attended human parainfluenza viruses from the influenza incidence surveillance project, 2010 2014. Pediatr Infect Dis J 35:717-722

12. Pan Y, Zhang Y, Shi W, Peng X, Cui S, Zhang D et al (2017) Human parainfluenza virus infection in severe acute respiratory infection cases in Beijing, 2014-2016: a molecular epidemiological study. Influenza Other Respir Viruses 11:564-568

13. Miall F, Rye A, Fraser M, Hunter A, Snowden JA (2002) Human parainfluenza type 4 infection: a case report highlighting pathogenicity and difficulties in rapid diagnosis in the post-transplant setting. Bone Marrow Transpl 29:541-542

14. Pawelczyk M, Kowalski ML (2017) The role of human parainfluenza virus infections in the immunopathology of the respiratory tract. Curr Allergy Asthma Rep 17:16

15. Essa S, Al-Tawalah H, AlShamali S, Al-Nakib W (2017) The potential influence of human parainfluenza viruses detected during hospitalization among critically ill patients in Kuwait, 2013-2015. Virol J 14:19

16. Canchola J, Vargosko AJ, Kim HW, Parrott RH, Christmas E, Jeffries B et al (1964) Antigenic variation among newly isolated strains of parainfluenza type 4 virus. Am J Hyg 79:357-364

17. Jansen RR, Schinkel J, Koekkoek S, Pajkrt D, Beld M, de Jong MD et al (2011) Development and evaluation of a four-tube real time multiplex PCR assay covering fourteen respiratory viruses, and comparison to its corresponding single target counterparts. J Clin Virol 51:179-185

18. Mao N, Ji Y, Xie Z, Wang H, Wang H, An J et al (2012) Human parainfluenza virus-associated respiratory tract infection among children and genetic analysis of HPIV-3 strains in Beijing, China. PLoS ONE 7:e43893

19. Frost HM, Robinson CC, Dominguez SR (2014) Epidemiology and clinical presentation of parainfluenza type 4 in children: a 3 -year comparative study to parainfluenza types $1-3$. J Infect Dis 209:695-702

20. Ren L, Gonzalez R, Xie Z, Xiong Z, Liu C, Xiang Z et al (2011) Human parainfluenza virus type 4 infection in Chinese children with lower respiratory tract infections: a comparison study. J Clin Virol 51:209-212

21. Vachon ML, Dionne N, Leblanc E, Moisan D, Bergeron MG, Boivin G (2006) Human parainfluenza type 4 infections, Canada. Emerg Infect Dis 12:1755-1758 
22. Billaud G, Morfin F, Vabret A, Boucher A, Gillet Y, Crassard N et al (2005) Human parainfluenza virus type 4 infections: a report of 20 cases from 1998 to 2002. J Clin Virol 34:48-51

23. Lau SK, Li KS, Chau KY, So LY, Lee RA, Lau YL et al (2009) Clinical and molecular epidemiology of human parainfluenza virus 4 infections in Hong Kong: subtype 4B as common as subtype 4A. J Clin Microbiol 47:1549-1552

24. Lau SK, To WK, Tse PW, Chan AK, Woo PC, Tsoi HW et al (2005) Human parainfluenza virus 4 outbreak and the role of diagnostic tests. J Clin Microbiol 43:4515-4521
Publisher's Note Springer Nature remains neutral with regard to jurisdictional claims in published maps and institutional affiliations. 